\title{
Combining Renal Cell Arrest and Damage Biomarkers to Predict Progressive AKI in Patient with Sepsis
}

\section{Xiaolei Tao}

Southern Medical University

Chunbo Chen

Southern Medical University

Weihong Luo

Southern Medical University

Jing Zhou

Southern Medical University

Jianwei Tian

Southern Medical University

xiaobing yang ( $\nabla$ yxb7829@163.com )

Southern Medical University

Fan Fan Hou

Southern Medical University

\section{Research}

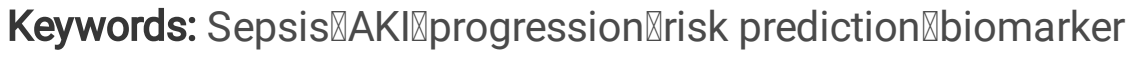

Posted Date: September 22nd, 2020

DOl: https://doi.org/10.21203/rs.3.rs-77423/v1

License: (9) This work is licensed under a Creative Commons Attribution 4.0 International License. Read Full License 
Combining renal cell arrest and damage biomarkers to predict progressive $\mathrm{AKI}$ in patient with sepsis

Xiaolei Tao ${ }^{1}$, Chunbo Chen ${ }^{1,2}$, Weihong Luo ${ }^{1}$, Jing Zhou ${ }^{1}$, Jianwei Tian ${ }^{1}$, Xiaobing Yang ${ }^{1}$, and Fan Fan $\mathrm{Hou}^{1}$

${ }^{1}$ Division of Nephrology, Nanfang Hospital, Southern Medical University, National Clinical Research Center for Kidney Disease, State Key Laboratory of Organ Failure Research, Guangzhou Regenerative Medicine and Health-Guangdong Laboratory, Guangzhou, China; ${ }^{2}$ Department of Critical Care Medicine, Guangdong Provincial People's Hospital, Guangdong Academy of Medical Sciences, 106 Zhongshan Er Road, Guangzhou, 510080, Guangdong, China.

Xiaolei Tao and Chunbo Chen contributed equally to this work

Running Title: biomarker predicts AKI progression in sepsis

Correspondence: Xiaobing Yang, MD and Fan Fan Hou PhD, Division of Nephrology, Nanfang Hospital, Southern Medical University, 1838 North Guangzhou Ave, Guangzhou 510515, China. Email: yxb7829@163.com and ffhouguangzhou@163.com

\section{Abstract word count: 328}

Text word count: 2730

Number of Tables and Figures: 7

Number of Supplementary Tables: 1 


\section{Abstract}

Background: Sepsis is the most common trigger for AKI and up to $40 \%$ of mild or moderate septic AKI would progress to more severe AKI, which is associated with significantly increased risk for death and later CKD/ESRD. Early identifying high risk patients for AKI progression is a major challenge in patients with septic AKI. This study aims to evaluated the utility of combining renal cell arrest and damage biomarkers for predicting the progression of AKI in patient with sepsis.

Methods: This is a prospective, multicenter cohort study which enrolled adult patients with sepsis and initially developed stage 1 or 2 AKI from January 2014 to March 2018. AKI was diagnosed and staged according to 2012 KDIGO-AKI guidelines. Renal cell arrest biomarkers (urinary TIMP2*IGFBP7, u[TIMP-2]*[IGFBP7]) and renal damage biomarkers (urinary KIM-1[uKIM-1] and urinary IL-18 [uIL-18]) were measured at time of AKI clinical diagnosis, and the utility of biomarkers for predicting septic AKI progression alone or in combination were evaluated. The primary outcome was AKI progression defined as worsening of AKI stage. The second outcome was AKI progression with subsequent death during hospitalization.

Results: Among 433 screened patients, 149 patients with sepsis and stage 1 or 2 AKI were included, in which 63 patients developed progressive AKI and 49 patients subsequently died during hospitalization. u[TIMP-2]*[IGFBP7], uKIM-1 and uIL-18 independently predicted the progression of septic AKI in which u[TIMP-2]*[IGFBP7] showed the greatest AUC $(0.745 ; 95 \% \mathrm{CI}, 0.667-0.823)$ as compared to uKIM-1 (AUC 0.719; 95\%CI 0.638-0.800) and uIL-18 (AUC 0.619; 95\%CI 0.5250.731). Combination of u[TIMP-2]*[IGFBP7] with uKIM-1 improved the performance of predicting septic AKI progression with AUC of 0.752. u[TIMP-2]*[IGFBP7], alone or combined with uKIM-1/uIL-18, improved the risk reclassification over the clinical risk factor model alone 
both for the primary and secondary outcomes, as evidenced by significant category-free net reclassification index.

Conclusion: Combination of renal cell arrest and damage biomarkers enhanced the prediction of AKI progression in patients with sepsis and improved risk reclassification over the clinical risk factors.

Keywords: Sepsis, AKI, progression, risk prediction, biomarker 


\section{Background}

Acute kidney injury (AKI) is a common complication in patients admitted to the intensive care unit (ICU), especially in those with sepsis [1]. Sepsis associated AKI accounts for approximately half of all AKI in ICU, which is associated with significantly increased risk for in-hospital death. Moreover, septic AKI is also associated with increased risk of later chronic kidney disease and end stage kidney disease [2].

AKI occurred in about $45-53 \%$ of patients with sepsis, and most septic AKI is mild or moderate AKI (KDIGO stage 1 or stage 2) [3,4]. However, study showed that up to $40 \%$ these mild or moderate AKI would progress to more severe AKI (KDIGO stage 3), of which 30\% required dialysis and the risk of death increased by 3 -fold, as high as $70 \%$ [5]. Therefore, early identifying patients at high risk for progressive AKI might help clinicians to enhance individualized monitoring and personalized management in patient with septic $\mathrm{AKI}$, which might prevent or halt the ongoing renal injury and improve the outcome of patients with sepsis.

Recently, there has been rising interest in searching and validating new biomarkers for early predicting AKI development and prognosis in different clinical settings. Renal cell cycle arrest biomarkers, urinary tissue inhibitor of metalloproteinases-2 (TIMP-2) and insulin-like growth factor binding protein-7 (IGFBP-7), have been shown to efficiently predict the risk of severe AKI development in ICU and were approved by U.S. FDA as a test of determine the risk of AKI development [6,7]. A recent study reported that urinary [TIMP-2]*[IGFBP7] concentration at the early phase of septic shock are independent factors to identify the population at high risk of progression from mild and moderate to severe AKI over the next 24 hours with an AUC of 0.83 [5]. In addition, there were other novel renal injury biomarkers, such as kidney injury molecular-1 (KIM- 
1) and interleukin-18 (IL-18), which reflecting different mechanism of AKI, were also shown to predict the progression of AKI in the setting of ICU and cardiac surgery, and presented modest performance $[8,9]$ To further improve the ability of biomarkers for predicting AKI progression in sepsis, carefully selecting and combining biomarkers might be a better approach for greater use. Compared with other AKI etiologies, septic AKI was thoughted to associated with multimechanisms, such as renal microcirculation disorder, renal cell cycle stress, tubular injury and inflammation $[1,7,10]$. Combining renal cell arrest biomarkers with renal injury/inflammation biomarkers to predict the progression of septic AKI was not addressed before, and whether combining renal cell arrest and damage biomarkers could improve risk classification for progressive AKI in sepsis warrants further investigation.

Here, we conducted a prospective, multicenter cohort study which included 149 adult septic patients who initially developed stage 1 or stage 2 AKI during ICU stay. Levels of novel urinary biomarkers ([TIMP-2]*[IGFBP7], KIM-1, and IL-18) were measured at time of AKI clinical diagnosis, and the utility of biomarkers for predicting septic AKI progression in combination were evaluate. Furthermore, the risk reclassification improvement of combining these biomarkers for predicting progressive septic AKI was investigated.

\section{Methods}

\section{Study Design and Study population}

We prospectively screened adult patients who were admitted to the ICU in two academic teaching hospitals in China from January 2014 to March 2018. Eligible participants were patients who admitted with sepsis and initially developed stage 1 or 2 AKI during hospitalization. The value of serum creatinine over a 6-month period before admission was used as baseline. Exclusion criteria included preexisting advanced CKD (baseline eGFR $<30 \mathrm{ml} / \mathrm{min}$ per $1.73 \mathrm{~m}^{2}$ ) and a life expectancy 
less than 24 hours. Patients with initial stage 3 AKI were excluded, since they would not progress further (Figure 1).

AKI was diagnosed according to the Kidney Disease Improving Global Outcomes (KDIGO) Clinical Practice Guidelines for AKI based on serum creatinine criteria [11]. Sepsis was defined according to The Third International Consensus Definitions for Sepsis and Septic Shock [12]. The study was approved by the Institutional Review Board of the National Clinical Research Center for Kidney Disease and all of the patients gave written informed consent.

\section{Procedures}

Spot urine samples were daily collected for the first 14 days during hospitalization. Urine samples at the day of AKI clinical diagnosis were used for biomarker measuring. Urine samples were centrifuged at $3000 \mathrm{rpm}$ for 10 minutes and the supernatants were stored at $-80^{\circ} \mathrm{C}$. Serum creatinine was measured on admission and twice a day during the first 5 days and at least daily thereafter. Clinical data for the study were collected from the hospital records, such as demographic, medication on admission, baseline renal function, Acute Physiology and Chronic Health Evaluation II (APACHE II) scores, Sequential Organ Failure Assessment (SOFA) scores, Multiple Organ Dysfunction Syndrome(MODS) scores, hemoglobin, blood urea nitrogen, serum albumin, blood lactate and procalcitonin.

\section{Laboratory Measurements}

All of the biomarkers were measured in a central laboratory using a standard protocol. All samples were labeled using study identification numbers without personal identifiers or clinical conditions. The levels of renal cell arrest biomarkers, urinary TIMP-2*IGFBP7 (u[TIMP-2]*[IGFBP7]), were measured by ELISA kits (TIMP-2: DTM200, R\&D Systems; IGFBP7: DY1334-05, R\&D Systems) according to the manufacturer's instructions. The levels of renal cell injury and inflammation biomarkers, urinary KIM-1 (uKIM-1) and urinary IL-18 (uIL-18), were measured by ELISA kits (KIM-1: DY1750B, R\&D Systems; IL-18: ELH-IL18, RayBiotech) on the manufacturer's instructions. All biomarkers were measured in triplicate. Investigator calculated intra- and interassay variability ranged $2 \%-6 \%$ and $5 \%-9 \%$ based on blinded replicate samples from study patients. 
Urinary albumin was measured using an automatic analyzer and reported as the ratio to urinary creatinine (UACR). All of the urinary biomarkers were normalized for urinary creatinine. Urinary creatinine was measured using an automatic biochemical analyzer. The eGFR was estimated by the CKD-Epidemiology Collaboration Equation [13]. Levels of biomarkers measured on the day of initial AKI clinical diagnosis were used for all analysis.

\section{Outcome Definitions}

The primary outcome was the progression of AKI, defined as worsening of KDIGO stage (from stage 1 to either stage 2 or stage 3, or from stage 2 to stage 3) as previously reported [14]. Patients treated with acute dialysis at any point during hospitalization were defined as stage 3 . The secondary outcome was AKI progression with death. Patients who died without progression were excluded from the primary analysis because death may have been a competing risk for progression for these patients.

\section{Statistical Analyses}

To compare continuous variables, we used a two-sample $t$ test or a Mann-Whitney U test. To compare categorical variables, we used the chi-squared or Fisher exact test. All tests were two-tailed and $\mathrm{P}<0.05$ was considered significant. To evaluate the performance of u[TIMP-2]*[IGFBP7] for predicting septic AKI progression, in single or in combination with renal damage biomarkers or clinical rias factors, we used the conventional area under the receiver-operating characteristic (ROC) curve (AUC). To evaluate the utility of renal arrest and damage biomarkers on risk classification, we determined the category-free net reclassification improvement (NRI) and the integrated discrimination improvement (IDI), as previously described $[15,16]$.

\section{Results}

\section{Cohort Characteristics}

A total of 433 patients admitted with sepsis in two hospitals were screened, and finally 149 patients with sepsis and stage 1 or 2 AKI were included for analysis (Figure 1). Among 149 septic patients with AKI, 
$79(53.0 \%)$ developed AKI on admission and 70 (47.0\%) during hospitalization.

Among 149 patients with stage 1 or 2 AKI, 63 patients (42.3\%) progressed to a higher stage of AKI during their hospitalization (32 individuals progressed to stage 2 and 31 progressed to stage 3); 23 of 63 (36.5\%) progressors received acute dialysis; 45 of $63(71.4 \%)$ developed AKI progression and subsequently died during hospitalization; 86 patients (57.7\%) persisted in stage 1 or 2 AKI.

The characteristics 149 septic patients with or without AKI progression are showed in Table 1. Compared to those with AKI that did not progress, patients with AKI progression had higher proportion of male, receiving vasoactive agents and antiarrhythmic agents on ICU admission. AKI progressors had higher score of illness severity, such as the APACHE II, SOFA, and MODS scores (Table 1). There was no statistical difference in age, baseline renal function, serum albumin, levels of blood lactate and procalcitonin, and proportion of morbidities (hypertension, diabetes, and pre-CKD) or receiving antibiotics on admission between patients with or without AKI progression.

Table 2 compares the characteristics at time of AKI diagnosis and the in-hospital outcomes between patients with or without AKI progression. Patients with AKI progression had a higher serum creatinine levels on the day of AKI diagnosis and a greater increase of serum creatinine levels from the baseline at time of AKI diagnosis. Patients with progressive AKI received higher proportion of vasopressors and antiarrhythmic agents. Levels of renal cell arrest biomarker (u[TIMP-2]*[IGFBP7]) and damage biomarkers (uKIM-1 and IL-18) were significantly higher in patients with AKI progression as compared to those without. Patients with AKI progression had more adverse outcomes, such as receiving acute dialysis and in-hospital death, as compared with those without AKI progression (Table 2). 
Performance of Combining u[TIMP-2]*[IGFBP7] and Renal Damage Biomarkers for Predicting Progressive AKI in Sepsis

Compared to those without AKI progression, patients with progressive AKI had significantly increased levels of u[TIMP-2]*[IGFBP7], uKIM-1, and uIL-18 at time of AKI clinical diagnosis (Table 2). As shown in Table S1, u[TIMP-2]*[IGFBP7], uKIM-1 and uIL-18 predicted the progression of AKI in sepsis, with u[TIMP-2]*[IGFBP7] presented the greatest AUC $(0.745,95 \%$ CI $0.667-0.823)$ as compared to uKIM-1 (AUC 0.719, 95\%CI 0.638-0.800) and uIL-18 (AUC 0.619, 95\%CI 0.525-0.713). For predicting AKI progression with death, u[TIMP-2]*[IGFBP7] also showed the greatest AUC (0.777, 95\%CI 0.700-0.854) as compared to uKIM-1 (AUC 0.738, 95\%CI 0.653-0.822), and uIL-18 (AUC 0.657, 95\%CI 0.557-0.758) (Table S1).

Combining renal cell arrest biomarker (u[TIMP-2]*[IGFBP7]) with renal damage biomarkers (uKIM-1 and uIL-18) improved the performance for predicting AKI progression, with AUCs of 0.752 for u[TIMP-2]*[IGFBP7] with uKIM-1, and 0.747 for u[TIMP-2]*[IGFBP7] with uIL-18, respectively (Table 3). For predicting AKI progression with death, combining u[TIMP-2]*[IGFBP7] with uKIM-1 produced an increased AUC of 0.782, as compare to u[TIMP-2]*[IGFBP7] alone. However, combining u[TIMP-2]*[IGFBP7] with uIL-18 could not improve the performance for predicting AKI progression with death as compare to u[TIMP-2]*[IGFBP7] alone. Combining u[TIMP-2]*[IGFBP7] with UACR could not further improve the performance both for predicting AKI progression or AKI progression with death in sepsis (Table 3).

Performance of Combining u[TIMP-2]*[IGFBP7] with Clinical Risk Factors for Predicting Progressive AKI in Sepsis 
Combining u[TIMP-2]*[IGFBP7] with clinical risk factors, such as APACHE II and SOFA score, serum creatinine and Cys-C at time of AKI diagnosis, improve the performance for predicting septic AKI progression and AKI progression with death (Table 4). The clinical risk factor model comprised of age, gender, APACHE II, serum creatinine and albuminuria at time of diagnosis predicts the primary and secondary outcomes with AUCs of 0.746 (95\%CI, 0.668-0.823) and 0.779 (95\%CI, 0.702-0.855), respectively (Figure 2). Combining u[TIMP-2]*[IGFBP7] with the clinical risk factor model further improved the AUCs to 0.797 (95\%CI, 0.726-0.867) and 0.845 (95\%CI, 0.780-0.910) as compared to clinical model alone both for predicting AKI progression or AKI progression with death. When combining both renal arrest and damage biomarkers with the clinical model, the predicting performance further improved, with AUCs of 0.806 (95\%CI, 0.738-0.874) and 0.846 (95\%CI, 0.780-0.910) for the primary and secondary outcomes (Table 4 and Figure 2).

Risk Classification Improvement of Combining u[TIMP-2]*[IGFBP7] with Renal Damage

\section{Biomarker or Clinical risk factors}

As shown in Table 5, adding u[TIMP-2]*[IGFBP7] to the clinical risk factor model resulted in the greatest improvement in risk reclassification both for the primary and the secondary outcomes, with a category-free net reclassification index (NRI) of 0.63 and 0.59 for AKI progression and AKI progression with death. Adding u[TIMP-2]*[IGFBP7] and uKIM-1 to the clinical risk factor model further improved risk classification over the clinical model alone, both for AKI progression and AKI progression with category-free NRI of 0.61 and 0.67 , respectively (Table 5).

\section{Discussion}


In this prospective, multicenter cohort study of adult patients with sepsis, we firstly showed that combining renal cell arrest biomarker and renal injury biomarkers could enhance the ability of biomarkers for predicting the progression of septic AKI. u[TIMP-2]*[IGFBP7], measured at time of AKI diagnosis, predicted both AKI progression and AKI progression with death in the setting of sepsis. Compared to u[TIMP-2]*[IGFBP7] alone, combination of u[TIMP-2]*[IGFBP7] with uKIM-1 slightly improved the performance for predicting both above outcomes, with AUC increased from 0.745 to 0.752 for AKI progression and from 0.777 to 0.782 for AKI progression with death. Moreover, we first showed that adding u[TIMP-2]*[IGFBP7] to the clinical risk factor model, alone or combined with renal injury biomarkers, significantly improved the risk classification of AKI progression and AKI progression with death in sepsis, as evidenced by significant NRI and IDI.

Sepsis is the most common trigger for AKI, septic patients were at the highest risk for developing AKI with an incidence ranged $22 \%-51 \%$ according to current $\operatorname{KDIGO} 2012$ criteria $[1,17,18]$. Patients who developed mild or moderate AKI and subsequently progressed to severe AKI had the highest risk for death [7]. In our cohort, near $80 \%$ of sepsis patients with progressive AKI died during hospitalization, consistent with previous reports. Therefore, using novel biomarkers to enhance the risk classification of AKI progression upon clinical risk factors might help clinicians initiate close patient monitoring and plan appropriate management, which in turn might reduce the risk of death of these patients based on above additional prognostic information. Previous studies have showed that renal arrest biomarkers, u[TIMP-2]*[IGFBP7], predicted the progression of AKI in the setting of ICU and septic shock [5,19-21]. Other novel renal injury or inflammation biomarkers, such as KIM-1, IL18, were also shown to predict progressive septic AKI [21-23], 
respectively. In this prospective study in patients with sepsis, we further directly compared the predictive performance of $\mathrm{u}[\mathrm{TIMP}-2]^{*}[$ [GFBP7] with the other novel injury/inflammation biomarkers in single or combination. Our results showed that combining u[TIMP-2]*[IGFBP7] with uKIM-1 could further improve the prediction of septic AKI progression compared to single biomarker prediction, which was also true for predicting AKI progression with death, suggesting that carefully selecting and combining biomarkers might be a better approach for greater application.

Albuminuria and serum creatinine are traditional markers of kidney injury, however, these existing markers have less sensitivity and specificity and are not sufficient for determining the risk of AKI progression [23-25]. Therefore, adding novel biomarkers to the clinical risk factor model which including albuminuria and serum creatinine would be a new way to increase risk assessment and stratification for AKI progression. The results of our study have showed that adding u[TIMP$2] *[$ IGFBP7] to the clinical risk factor model could significantly improve risk classification for AKI progression alone or in combination with uKIM-1, with NRIs of 0.63 and 0.61 respectively. And this was also true for risk classification for the secondary outcome, i.e. AKI progression with death, with NRIs of 0.59 and 0.67. u[TIMP-2]*[IGFBP7], measured at time of septic AKI diagnosis, could not only be used as a tool assessing the risk of AKI progression in sepsis, but also provided additional prognostic information in hospital, such as subsequent death after AKI. Interestingly, combining u[TIMP-2]*[IGFBP7] with uKIM-1 and uIL-18 together could not significantly improve prediction of septic AKI progression as compared to u[TIMP-2]*[IGFBP7] with uKIM-1 combination, suggesting that efficiently selecting and combining biomarkers for a multi-biomarker approach prediction might need more investigation.

\section{Strengths and limitations}


Our study has the following strength. First, this is a multicenter, prospective cohort study. AKI and sepsis were diagnosed based on standardized criteria (KDIGO 2012 and sepsis-3) that are currently used in the international renal and critical care community. Second, serum creatinine was measured daily to precisely define AKI and determine AKI progression. Third, we simultaneously measured well reported renal cell arrest biomarker and renal damage biomarkers and assessed the predictive performance and risk classification alone or combination with clinical risk factors in the setting of sepsis, which directly compares the predictive ability of biomarkers alone or in combination. This study also had limitations. Urinary creatinine excretion is not at a steady state during AKI; $24 \mathrm{~h}$ urinary excretion of biomarkers would be more meaningful. The number of primary outcomes was relatively small, and all patients were Chinese adults; validation studies from other ethnic populations are warranted.

\section{Conclusion}

In conclusion, combination of renal arrest and damage biomarkers enhanced the prediction of AKI progression in patient with sepsis and improved risk reclassification over the clinical risk factor model alone.

\section{Acknowledgements}

Not applicable.

\section{Authors' contributions}

Dr. Yang and Dr. Hou designed the study. Dr. Tao, Dr. Chen, Dr. Luo, Dr. Zhou, and Dr. Tian performed data collection and sample measurements. Dr. Tao and Dr. Yang analyzed the data. Dr. 
Tao and Dr. Yang drafted and revised the paper. All authors approved the final manuscript as submitted.

\section{Funding}

This research was supported by the National Natural Science Foundation of China (81670636 and 81970666 to XBY); the President Foundation of Nanfang Hospital (2018Z019 to XBY), Southern Medical University; and the Outstanding Youths Development Scheme of Nanfang Hospital (2018J001 to XBY), Southern Medical University.

\section{Availability of data and materials}

Privacy or ethical restrictions apply to the availability of these data. Thus, these data are not publicly available, however, are available from the authors upon reasonable request.

\section{Disclosures}

The authors declare that there is no conflict of interests.

\section{Ethics approval and consent to participate}

The study was approved by the Institutional Review Board of the National Clinical Research Center for Kidney Disease and all of the patients gave written informed consent.

\section{Consent for publication}

Not applicable. 


\section{Competing interests}

The authors declare that they have no competing interests. 


\section{References}

1. Bellomo R, Kellum JA, Ronco C, Wald R, Martensson J, Maiden M, et al. Acute kidney injury in sepsis. Intensive Care Med. 2017;43(6):816-28.

2. Coca SG, Singanamala S, Parikh CR. Chronic kidney disease after acute kidney injury: a systematic review and meta-analysis. Kidney Int. 2012;81(5):442-8.

3. Gordon AC, Mason AJ, Thirunavukkarasu N, Perkins GD, Cecconi M, Cepkova M, et al. Effect of early vasopressin vs norepinephrine on kidney failure in patients with septic shock: The VANISH Randomized Clinical Trial. JAMA. 2016;316(5):509-18.

4. Poukkanen M, Vaara ST, Pettila V, Kaukonen KM, Korhonen AM, Hovilehto S, et al. Acute kidney injury in patients with severe sepsis in Finnish Intensive Care Units. Acta Anaesthesiol Scand. 2013;57(7):863-72.

5. Maizel J, Daubin D, Vong LV, Titeca-Beauport D, Wetzstein M, Kontar L, et al. Urinary TIMP2 and IGFBP7 identifies high risk patients of short-term progression from mild and moderate to severe acute kidney injury during septic shock: a prospective cohort study. Dis Markers. 2019; 347:1215.

6. Gunnerson KJ, Shaw AD, Chawla LS, Bihorac A, Al-Khafaji A, Kashani K, et al. TIMP2*IGFBP7 biomarker panel accurately predicts acute kidney injury in high-risk surgical patients. J Trauma Acute Care Surg. 2016;80(2):243-9.

7. Kashani K, Al-Khafaji A, Ardiles T, Artigas A, Bagshaw SM, Bell M, et al. Discovery and validation of cell cycle arrest biomarkers in human acute kidney injury. Crit Care. 2013;17(1): R25.

8. Koyner JL, Garg AX, Coca SG, Sint K, Thiessen-Philbrook H, Patel UD, et al. Biomarkers predict progression of acute kidney injury after cardiac surgery. J Am Soc Nephrol. 2012;23(5):90514.

9. Parr SK, Clark AJ, Bian A, Shintani AK, Wickersham NE, Ware LB, et al. Urinary L-FABP predicts poor outcomes in critically ill patients with early acute kidney injury. Kidney Int. 2015;87(3):640-8.

10. Gomez H, Ince C, De Backer D, Pickkers P, Payen D, Hotchkiss J, et al. A unified theory of sepsis-induced acute kidney injury: inflammation, microcirculatory dysfunction, bioenergetics, and the tubular cell adaptation to injury. Shock. 2014;41(1):3-11.

11. KDIGO Acute Kidney Injury Work Group (2012). KDIGO clinical practice guideline for acute kidney injury. Kidney Int Suppl. 2013;(2):1-138.

12. Singer M, Deutschman CS, Seymour CW, Shankar-Hari M, Annane D, Bauer M, et al. The Third International Consensus Definitions for Sepsis and Septic Shock (Sepsis-3). JAMA. 2016;315(8):801-10.

13. Levey SA, Stevens AL, Schmid HC, Zhang YP, Castro FA, Feldman IH, et al for the Chronic Kidney Disease Epidemiology Collaboration (CKD-EPI). A new equation to estimate glomerular filtration rate. Ann Intern Med. 2009;150(9):604-12.

14. Chen C, Yang X, Lei Y, Zha Y, Liu H, Ma C, et al. Urinary biomarkers at the time of AKI diagnosis as predictors of progression of AKI among patients with acute cardiorenal syndrome. Clin J Am Soc Nephrol. 2016;11(9):1536-44.

15. Cook NR. Statistical evaluation of prognostic versus diagnostic models: beyond the ROC curve. Clin Chem. 2008;54(1):17-23.

16. Pencina MJ, D'Agostino RB, Sr., D'Agostino RB, Jr., Vasan RS. Evaluating the added predictive ability of a new marker: from area under the ROC curve to reclassification and beyond. Stat Med. 2008;27(2):157-72.

17. Angus CD, Linde-Zwirble TW, Lidicker J, Clermont G, Carcillo J, Pinsky RM. Epidemiology of severe sepsis in the United States analysis of incidence, outcome, and associated costs of care. Crit Care Med. 2001; 29:1303-10.

18. Vincent JL, Sakr Y, Sprung CL, Ranieri VM, Reinhart K, Gerlach H, et al. Sepsis in European intensive care units: results of the SOAP study. Crit Care Med. 2006;34(2):344-53.

19. Honore PM, Nguyen HB, Gong M, Chawla LS, Bagshaw SM, Artigas A, et al. Urinary tissue inhibitor of metalloproteinase-2 and insulin-like growth factor-binding protein 7 for risk stratification of acute kidney injury in patients with sepsis. Crit Care Med. 2016;44(10):1851-60.

20. Ferrari F, Romero-Gonzalez G, Topete LR, Senzolo M, Lorenzin A, Husain-Syed F, et al. Routine adoption of urinary [IGFBP7]*[TIMP-2] to assess acute kidney injury at any stage 12 hours after intensive care unit admission: a prospective cohort study. Sci Rep. 2019;9(1):16484.

21. Fiorentino M, Xu ZY, Smith A, Singbartl K, Palevsky MP, Chawla SL, et al for the ProCESS and ProGReSS-AKI Investigators. Serial measurement of cell-cycle arrest biomarkers [TIMP- 
2]•[IGFBP7] and risk for progression to death, dialysis or severe acute kidney injury in patients with septic shock. Am J Respir Crit Care Med. 2020.

22. Fan H, Zhao Y, Sun M, Zhu JH. Urinary neutrophil gelatinase-associated lipocalin, kidney injury molecule-1, N-acetyl-beta-D-glucosaminidase levels and mortality risk in septic patients with acute kidney injury. Arch Med Sci. 2018;14(6):1381-6.

23. Urbschat A, Obermuller N, Haferkamp A. Biomarkers of kidney injury. Biomarkers. 2011;16 Suppl 1: S22-30.

24. Star RA. Treatment of acute renal failure. Kidney Int. 1998; 54:1817-31.

25. Nguyen MT, Devarajan P. Biomarkers for the early detection of acute kidney injury. Pediatr Nephrol. 2008;23(12):2151-7. 


\section{Figure Legends}

Figure 1. Flow chart of patient enrollment and exclusion.

Figure 2. ROC analyses for predicting AKI progression or AKI progression with death.

(A) The AUCs of renal cell arrest and damage biomarkers (uTIMP2*IGFBP7 and uKIM-1), and clinical model, at the time of AKI diagnosis, for predicting AKI progression. (B) The AUCs of renal cell arrest and damage biomarkers (uTIMP2*IGFBP7 and uKIM-1), and clinical model, at the time of AKI diagnosis, for predicting AKI progression with subsequent death. 
Table 1 Characteristics of septic patients with and without AKI progression

\begin{tabular}{|c|c|c|c|c|}
\hline Characteristics & Overall $(n=149)$ & $\begin{array}{l}\text { Progression } \\
\quad(\mathrm{n}=63)\end{array}$ & $\begin{array}{c}\text { Non-Progression } \\
\quad(\mathrm{n}=86)\end{array}$ & $P$ \\
\hline \multicolumn{5}{|l|}{ Demographics } \\
\hline Age, y & $59.6 \pm 16.0$ & $60.8 \pm 15.2$ & $58.8 \pm 16.6$ & 0.536 \\
\hline Male, n (\%) & $99(66.4)$ & $34(54.0)$ & $65(75.6)$ & 0.006 \\
\hline Hypertension, n (\%) & $67(45.0)$ & $28(44.4)$ & $39(45.3)$ & 0.913 \\
\hline Diabetes, $\mathrm{n}(\%)$ & $34(22.8)$ & $15(23.8)$ & $19(22.1)$ & 0.805 \\
\hline Prior $\mathrm{CKD}^{\mathrm{a}}, \mathrm{n}(\%)$ & $15(10.1)$ & $8(12.7)$ & $7(8.1)$ & 0.361 \\
\hline \multicolumn{5}{|l|}{ Medication on ICU admission } \\
\hline Antibiotics, n (\%) & $105(70.5)$ & $40(63.5)$ & $65(75.6)$ & 0.110 \\
\hline Vasopressors, n (\%) & $19(12.8)$ & $13(20.6)$ & $6(7.0)$ & 0.014 \\
\hline Antiarrhythmic agents, $\mathrm{n}(\%)$ & $7(4.7)$ & $6(9.5)$ & $1(1.2)$ & 0.017 \\
\hline \multicolumn{5}{|l|}{ Baseline renal function } \\
\hline Serum creatinine, mg/dL & $0.9 \pm 0.3$ & $0.9 \pm 0.3$ & $0.9 \pm 0.3$ & 0.861 \\
\hline eGFR, $\mathrm{ml} / \mathrm{min}$ per $1.73 \mathrm{~m}^{2}$ & $90.7 \pm 27.0$ & $87.8 \pm 28.5$ & $91.7 \pm 25.9$ & 0.429 \\
\hline \multicolumn{5}{|l|}{ Parameters on ICU admission } \\
\hline APACHE II & $20.0(13.0-25.0)$ & $23.0(18.0-27.0)$ & $17.0(11.8-23.0)$ & $<0.001$ \\
\hline SOFA & $6.0(4.0-8.0)$ & $7.0(5.0-9.0)$ & $5.0(4.0-7.0)$ & 0.031 \\
\hline MODS & $5.0(3.0-6.0)$ & $5.0(4.0-7.0)$ & $4.0(3.0-6.0)$ & 0.033 \\
\hline Hemoglobin, $g / L$ & $118.1 \pm 78.1$ & $106.7 \pm 30.4$ & $126.5 \pm 98.8$ & 0.014 \\
\hline Blood urea nitrogen, $\mathrm{mmol} / \mathrm{L}$ & $9.6 \pm 7.4$ & $10.6 \pm 8.0$ & $8.9 \pm 6.9$ & 0.274 \\
\hline Serum albumin, $\mathrm{g} / \mathrm{L}$ & $29.3 \pm 7.7$ & $28.2 \pm 6.9$ & $30.1 \pm 8.1$ & 0.072 \\
\hline Blood lactate, $\mathrm{mmol} / \mathrm{L}$ & $2.9 \pm 2.1$ & $3.3 \pm 2.2$ & $2.7 \pm 2.0$ & 0.086 \\
\hline Procalcitonin, ng/ml & $2.2(0.2-19.8)$ & $2.1(0.9-9.0)$ & $2.6(0.1-41.3)$ & 0.900 \\
\hline
\end{tabular}

Continuous variables were expressed as mean $\pm \mathrm{SD}$ or median $\left(25^{\text {th }}\right.$ percentile- $75^{\text {th }}$ percentile, interquartile range). Categorical variables were expressed as a number (\%).

AKI progression is defined as worsening of AKI stage.

a Defined as baseline eGFR $<60 \mathrm{ml} / \mathrm{min}$ per $1.73 \mathrm{~m}^{2}$. Baseline eGFR was calculated by CKDEpidemiology Collaboration equation according to at least three measurements of serum creatinine over a 6-month period before admission.

Abbreviation: AKI, acute kidney injury; CKD, chronic kidney disease; ICU, intensive care unit; eGFR, estimated glomerular filtration rate; APACHE II, Acute Physiology and Chronic Health Evaluation II; SOFA, Sequential Organ Failure Assessment; MODS, Multiple Organ Dysfunction Syndrome. 
Table 2 Characteristics at time of AKI diagnosis in septic patients with and without AKI progression

\begin{tabular}{|c|c|c|c|c|}
\hline Characteristics & Overall $(n=149)$ & $\begin{array}{l}\text { Progression } \\
\qquad(\mathrm{n}=63)\end{array}$ & $\begin{array}{l}\text { Non-Progression } \\
\qquad(\mathrm{n}=86)\end{array}$ & $P$ \\
\hline \multicolumn{5}{|l|}{ AKI Severity } \\
\hline $\mathrm{SCr}$ at $\mathrm{AKI}$ diagnosis, $\mathrm{mg} / \mathrm{dL}$ & $1.6 \pm 0.5$ & $1.7 \pm 0.5$ & $1.5 \pm 0.5$ & 0.022 \\
\hline Peak SCr, mg/dL & $1.8 \pm 0.8$ & $2.2 \pm 0.9$ & $1.6 \pm 0.5$ & $<0.001$ \\
\hline Change in $\mathrm{SCr}^{\mathrm{a}}, \mathrm{mg} / \mathrm{dL}$ & $1.0 \pm 0.6$ & $1.3 \pm 0.7$ & $0.7 \pm 0.4$ & $<0.001$ \\
\hline SCys-C at AKI diagnosis, $\mathrm{mg} / \mathrm{L}$ & $1.5 \pm 0.7$ & $1.7 \pm 0.7$ & $1.3 \pm 0.7$ & 0.004 \\
\hline AKI duration, $\mathrm{d}$ & $2.0(1.0-4.0)$ & $3.5(2.0-5.0)$ & $1.0(1.0-3.0)$ & $<0.001$ \\
\hline \multicolumn{5}{|c|}{ Medications at time of AKI diagnosis } \\
\hline Vasopressors, $\mathrm{n}(\%)$ & $79(53.0)$ & $52(82.5)$ & $27(31.4)$ & $<0.001$ \\
\hline Antiarrhythmic agents, $\mathrm{n}(\%)$ & $19(12.8)$ & $16(25.4)$ & $3(3.5)$ & $<0.001$ \\
\hline Blood transfusion, $\mathrm{n}(\%)$ & $136(91.3)$ & $61(96.8)$ & $75(87.2)$ & 0.040 \\
\hline Mechanical ventilation, $\mathrm{n}(\%)$ & $135(90.6)$ & $59(93.7)$ & $76(88.4)$ & 0.275 \\
\hline \multicolumn{5}{|c|}{ Biomarkers at time of AKI diagnosis } \\
\hline $\mathrm{u}[\mathrm{TIMP}-2]^{*}[\mathrm{IGFBP} 7],(\mu \mathrm{g} / \mathrm{g} \mathrm{Cr})^{2}$ & $\begin{array}{c}1169.7 \\
(426.6-3079.8)\end{array}$ & $\begin{array}{c}2168.5 \\
(1068.8-5274.9)\end{array}$ & $\begin{array}{c}583.2 \\
(293.6-1666.4)\end{array}$ & $<0.001$ \\
\hline $\mathrm{uKIM}-1, \mu \mathrm{g} / \mathrm{g} \mathrm{Cr}$ & $3.1(1.5-6.0)$ & $5.0(2.7-7.3)$ & $2.1(0.8-4.8)$ & $<0.001$ \\
\hline uIL-18, ng/g Cr & $\begin{array}{c}196.5 \\
(79.5-664.5)\end{array}$ & $\begin{array}{c}384.4 \\
(89.4-1228.4)\end{array}$ & $\begin{array}{c}131.5 \\
(70.9-433.7)\end{array}$ & 0.017 \\
\hline $\mathrm{uACR}, \mathrm{mg} / \mathrm{g} \mathrm{Cr}$ & $\begin{array}{c}138.3 \\
(47.8-476.5)\end{array}$ & $\begin{array}{c}221.5 \\
(76.6-546.2)\end{array}$ & $\begin{array}{c}108.8 \\
(33.9-302.3)\end{array}$ & 0.006 \\
\hline \multicolumn{5}{|l|}{ In-hospital outcomes } \\
\hline ICU stay, d & $7.0(4.0-12.0)$ & $7.0(4.5-12.0)$ & $6.0(3.0-12.0)$ & 0.296 \\
\hline Acute dialysis, $\mathrm{n}(\%)$ & $23(15.4)$ & $23(36.5)$ & $0(0.0)$ & $<0.001$ \\
\hline In-hospital death, $n(\%)$ & $45(30.2)$ & $45(71.4)$ & $0(0.0)$ & $<0.001$ \\
\hline
\end{tabular}

AKI progression is defined as worsening of AKI stage.

${ }^{\text {a }}$ Serum creatinine level on the day of AKI diagnosis minus baseline serum creatinine level.

Abbreviation: SCr, serum creatinine; SCys-C, serum cystatin C. u[TIMP-2]*[IGFBP7], urinary tissue inhibitor of metalloproteinase-2 and insulin-like growth factor-binding protein 7; uKIM-1, urinary kidney injury moleculer-1; uIL-18, urinary Interleukin-18; uACR, urinary albumin to creatinine ratio. 
Table 3 Performance of renal arrest biomarkers for predicting septic AKI progression or AKI progression with death in single or combination with renal damage biomarkers

\begin{tabular}{llc}
\hline Outcomes & AUC & $\mathbf{9 5 \%}$ CI \\
\hline AKI progression & & \\
u[TIMP-2]*[IGFBP7] & 0.745 & 0.667 to 0.823 \\
u[TIMP-2]*[IGFBP7] +uKIM-1 & 0.752 & 0.675 to 0.828 \\
u[TIMP-2]*[IGFBP7]+uIL-18 & 0.747 & 0.669 to 0.825 \\
u[TIMP-2]*[IGFBP7]+uACR & 0.745 & 0.668 to 0.823 \\
u[TIMP-2]*[IGFBP7]+uKIM-1+uIL-18+uACR & 0.755 & 0.679 to 0.832 \\
AKI progression with death & & \\
u[TIMP-2]*[IGFBP7] & 0.777 & 0.700 to 0.854 \\
u[TIMP-2]*[IGFBP7]+uKIM-1 & 0.782 & 0.705 to 0.859 \\
u[TIMP-2]*[IGFBP7]+uIL-18 & 0.777 & 0.700 to 0.854 \\
u[TIMP-2]*[IGFBP7]+uACR & 0.778 & 0.700 to 0.855 \\
u[TIMP-2]*[IGFBP7]+uKIM-1+uIL-18+uACR & 0.780 & 0.703 to 0.857 \\
\hline
\end{tabular}

AKI progression is defined as worsening of AKI stage.

Abbreviation: AUC, area under the receiver-operating characteristic curve; $\mathrm{CI}$, confidence interval; u[TIMP-2]*[IGFBP7], urinary tissue inhibitor of metalloproteinase-2 and insulin-like growth factor-binding protein 7; uKIM-1, urinary kidney injury moleculer-1; uIL-18, urinary Interleukin18 ; uACR, urinary albumin to creatinine ratio. 
Table 4 Performance of renal arrest biomarkers for predicting septic AKI progression in single or combination with clinical risk factors

\begin{tabular}{|c|c|c|}
\hline Outcomes & AUC & $95 \% \mathrm{CI}$ \\
\hline \multicolumn{3}{|l|}{ AKI progression } \\
\hline $\mathrm{u}[\mathrm{TIMP}-2] *[$ IGFBP7] & 0.745 & 0.667 to 0.823 \\
\hline u[TIMP-2]*[IGFBP7]+APACHE II & 0.779 & 0.706 to 0.852 \\
\hline $\mathrm{u}[\mathrm{TIMP}-2] *[$ IGFBP7]]+SOFA & 0.752 & 0.675 to 0.829 \\
\hline $\mathrm{u}[\mathrm{TIMP}-2] *[$ IGFBP7] $+\mathrm{SCr}$ at time of AKI diagnosis & 0.752 & 0.675 to 0.829 \\
\hline $\mathrm{u}[\mathrm{TIMP}-2] *[$ IGFBP7]+SCys-C at time of AKI diagnosis & 0.754 & 0.677 to 0.831 \\
\hline $\mathrm{u}[\mathrm{TIMP}-2] *\left[\right.$ IGFBP7] $+\mathrm{M}^{\mathrm{a}}$ & 0.797 & 0.726 to 0.867 \\
\hline $\mathrm{u}[\mathrm{TIMP}-2]^{*}[\mathrm{IGFBP} 7]+\mathrm{uKIM}-1+\mathrm{M}$ & 0.806 & 0.738 to 0.874 \\
\hline \multicolumn{3}{|l|}{ AKI progression with death } \\
\hline u[TIMP-2]*[IGFBP7] & 0.777 & 0.700 to 0.854 \\
\hline u[TIMP-2]*[IGFBP7]+APACHE II & 0.828 & 0.760 to 0.897 \\
\hline $\mathrm{u}[\mathrm{TIMP}-2]^{*}[$ IGFBP7]]+SOFA & 0.797 & 0.723 to 0.871 \\
\hline $\mathrm{u}[\mathrm{TIMP}-2]^{*}[\mathrm{IGFBP}]+\mathrm{SCr}$ at time of AKI diagnosis & 0.784 & 0.708 to 0.860 \\
\hline $\mathrm{u}[\mathrm{TIMP}-2]^{*}[$ IGFBP7]+SCys-C at time of AKI diagnosis & 0.785 & 0.708 to 0.861 \\
\hline $\mathrm{u}[\mathrm{TIMP}-2] *[$ IGFBP7] $+\mathrm{M}$ & 0.845 & 0.780 to 0.910 \\
\hline $\mathrm{u}[\mathrm{TIMP}-2] *[$ IGFBP7]+uKIM-1+M & 0.846 & 0.780 to 0.910 \\
\hline
\end{tabular}

AKI progression is defined as worsening of AKI stage.

${ }^{a} \mathrm{M}$, clinical risk model. The clinical risk model for predicting AKI progression are comprised of age, gender, APACHE II, SCr at time of diagnosis, uACR at time of AKI diagnosis (AUC $0.746,95 \%$ CI 0.668 to 0.823 ); The clinical risk model for predicting AKI progression with death are comprised of age, gender, APACHE II, SCr at time of diagnosis, uACR at time of AKI diagnosis (AUC $0.779,95 \%$ CI 0.702 to 0.855 )

\section{Abbreviation:}

AUC, area under the receiver-operating characteristic curve; $\mathrm{CI}$, confidence interval; u[TIMP2]*[IGFBP7], urinary tissue inhibitor of metalloproteinase-2 and insulin-like growth factorbinding protein 7; APACHE II, Acute Physiology and Chronic Health Evaluation; SOFA, Sequential Organ Failure Assessment; SCr, serum creatinine; SCys-C, serum cystatin C; uKIM-1, urinary kidney injury moleculer-1. 
Table 5 Analysis of risk reclassification of biomarkers over the clinical model for predicting AKI progression or AKI progression with death

\begin{tabular}{|c|c|c|c|c|c|c|c|c|}
\hline Outcomes & $\begin{array}{l}\text { Category-Free } \\
\text { NRI }(95 \% \mathrm{CI})\end{array}$ & $P$ & $\begin{array}{l}\text { NRI in Progressors } \\
\quad(95 \% \mathrm{CI})\end{array}$ & $P$ & $\begin{array}{l}\text { NRI in Non-progressors } \\
(95 \% \mathrm{CI})\end{array}$ & $P$ & IDI $(95 \% \mathrm{CI})$ & $P$ \\
\hline \multicolumn{9}{|l|}{ AKI progression } \\
\hline Clinical risk factors & referent & & referent & & referent & & referent & \\
\hline $\begin{array}{l}\text { Clinical risk factors }+u \text { [TIMP- } \\
2]^{*}[\text { IGFBP7 }]\end{array}$ & $0.63(0.31-0.94)$ & $<0.001$ & $0.30(0.06-0.54)$ & 0.015 & $0.33(0.12-0.53)$ & 0.002 & $0.08(0.04-0.13)$ & $<0.001$ \\
\hline Clinical risk factors $+\mathrm{uKIM}-1$ & $0.53(0.21-0.86)$ & 0.001 & $0.30(0.06-0.54)$ & 0.015 & $0.23(0.02-0.44)$ & 0.030 & $0.09(0.04-0.14)$ & $<0.001$ \\
\hline Clinical risk factors $+\mathrm{uIL}-18$ & $0.31(-0.02-0.64)$ & 0.059 & $0.14(-0.11-0.39)$ & 0.260 & $0.16(-0.05-0.38)$ & 0.132 & $0.02(0.00-0.05)$ & 0.048 \\
\hline $\begin{array}{l}\text { Clinical risk factors }+u[\text { TIMP- } \\
2]^{*}[\text { IGFBP7] }+u K I M-1\end{array}$ & $0.61(0.29-0.93)$ & $<0.001$ & $0.33(0.09-0.57)$ & 0.007 & $0.28(0.07-0.49)$ & 0.009 & $0.11(0.05-0.16)$ & $<0.001$ \\
\hline \multicolumn{9}{|l|}{ AKI progression with death } \\
\hline Clinical risk factors & referent & & referent & & referent & & referent & \\
\hline $\begin{array}{l}\text { Clinical risk factors }+\mathrm{u} \text { [TIMP- } \\
2]^{*}[\text { IGFBP7] }\end{array}$ & $0.59(0.25-0.94)$ & $<0.001$ & $0.27(-0.01-0.54)$ & 0.063 & $0.33(0.12-0.53)$ & 0.002 & $0.12(0.06-0.18)$ & $<0.001$ \\
\hline Clinical risk factors $+\mathrm{uKIM}-1$ & $0.56(0.21-0.90)$ & 0.002 & $0.35(0.08-0.62)$ & 0.014 & $0.21(0.00-0.42)$ & 0.052 & $0.10(0.05-0.16)$ & $<0.001$ \\
\hline Clinical risk factors $+\mathrm{uIL}-18$ & $0.50(0.15-0.85)$ & 0.002 & $0.22(-0.06-0.51)$ & 0.117 & $0.28(0.07-0.49)$ & 0.009 & $0.05(0.01-0.10)$ & 0.006 \\
\hline $\begin{array}{l}\text { Clinical risk factors }+u[\text { TIMP- } \\
2]^{*}[\text { IGFBP7] }+u K I M-1\end{array}$ & $0.67(0.33-1.01)$ & $<0.001$ & $0.35(0.08-0.62)$ & 0.014 & $0.33(0.12-0.53)$ & 0.002 & $0.13(0.07-0.19)$ & $<0.001$ \\
\hline
\end{tabular}

AKI progression is defined as worsening of AKI stage.

Clinical risk factors for AKI progression are comprised of age, gender, APACHE II, SCr at time of diagnosis, uACR at time of AKI diagnosis. 
Abbreviation:

NRI, net reclassification improvement; IDI, integrated discrimination improvement. $\mathrm{SCr}$, serum creatinine; uACR, urinary albumin to creatinine ratio. 


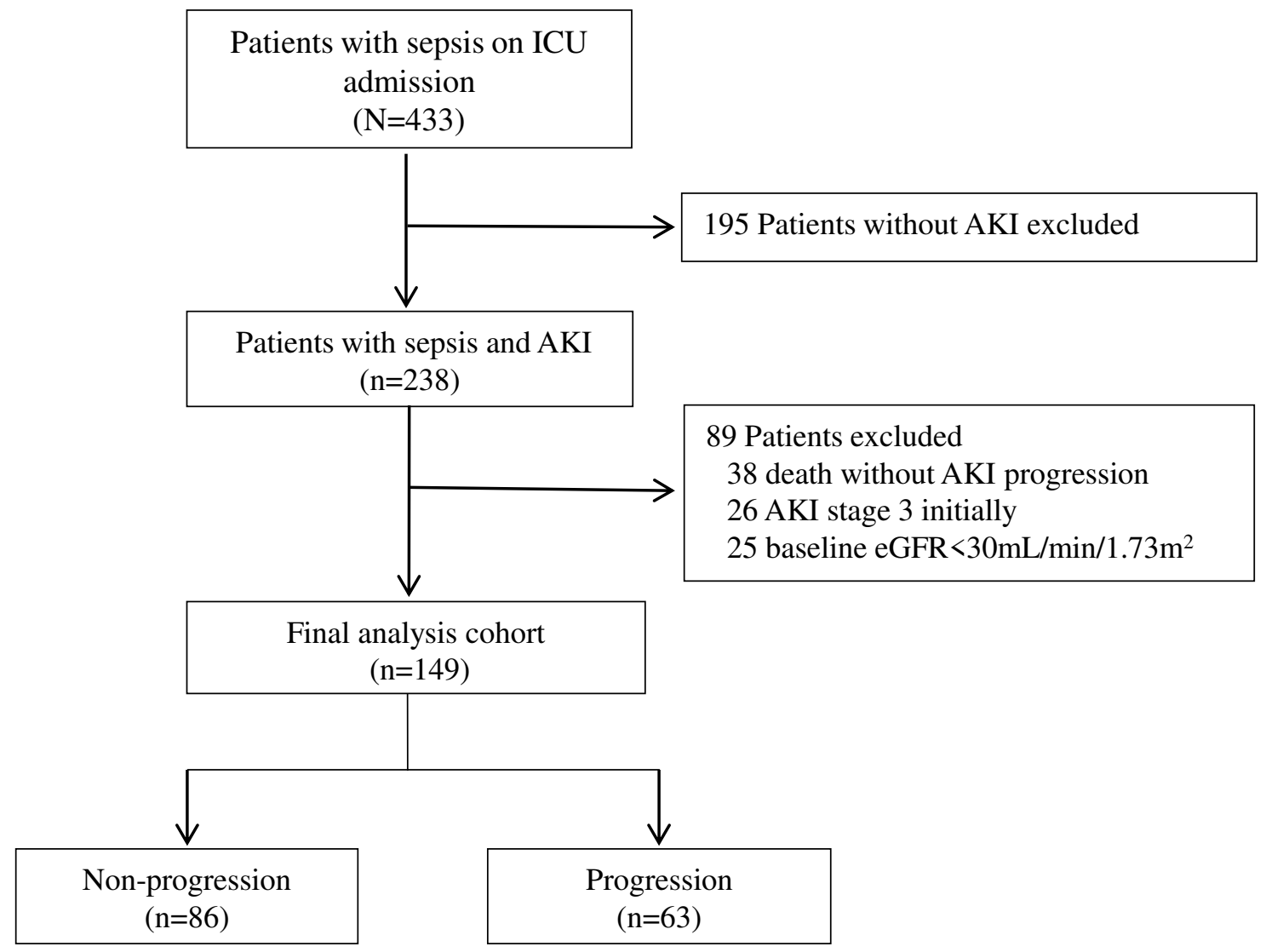

Fig. 1 Flow chart of patient enrollment and exclusion. 

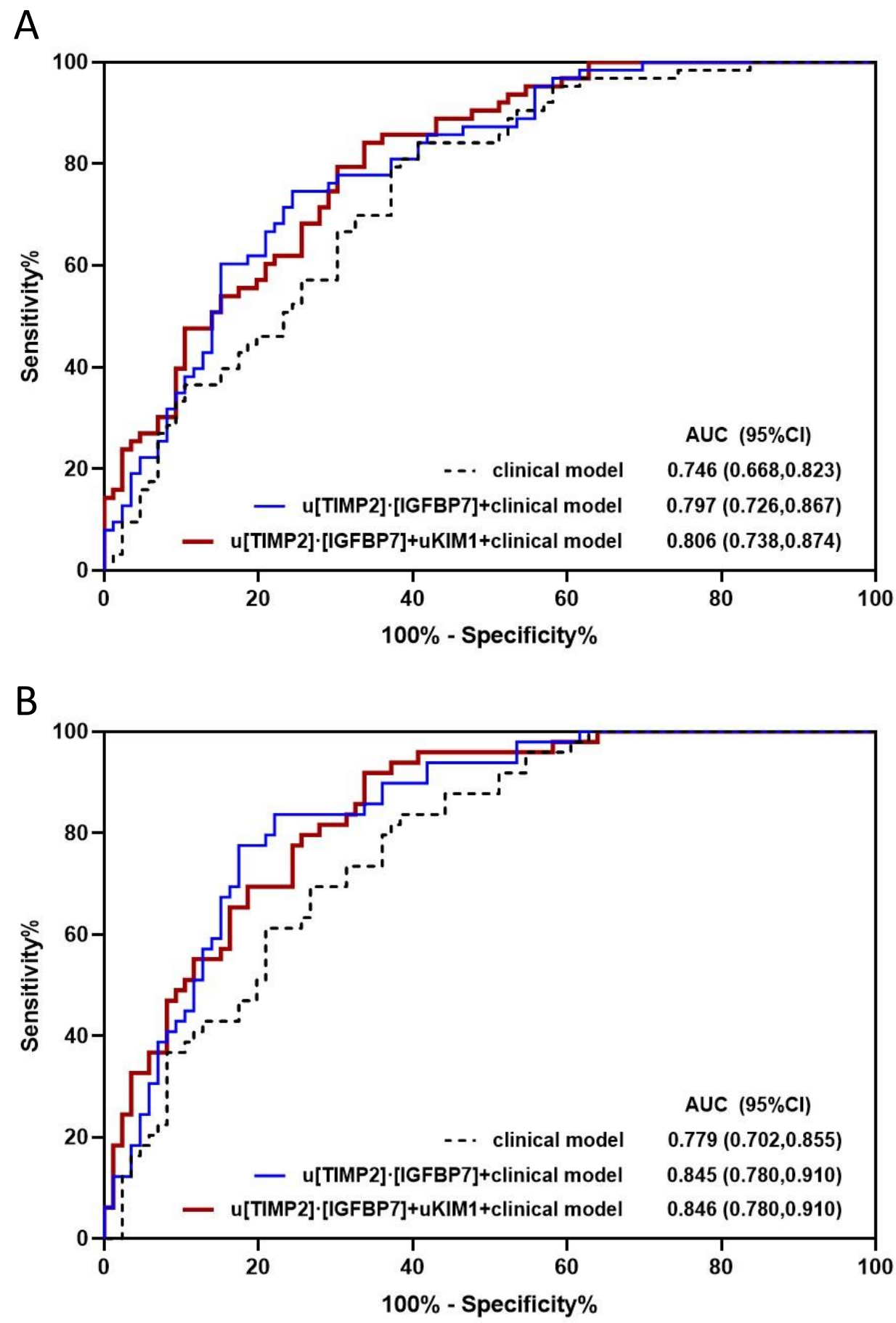

Fig. 2 ROC analyses for predicting AKI progression or AKI progression with death

(A) The AUCs of renal cell arrest and damage biomarkers (uTIMP2*IGFBP7 and uKIM-1), and clinical model, at the time of AKI diagnosis, for predicting AKI progression. (B) The AUCs of renal cell arrest and damage biomarkers (uTIMP2*IGFBP7 and uKIM1), and clinical model, at the time of AKI diagnosis, for predicting AKI progression with subsequent death. 


\section{Supplemental Material}

Table S1. Performance of renal cell arrest and damage biomarkers for predicting septic AKI progression or AKI progression with death

$\underline{\text { Outcomes }}$

\section{AKI progression}

u[TIMP-2]*[IGFBP7]

uKIM-1

uIL-18

uACR

AKI progression with death
AUC

0.745

0.719

0.619

0.631

0.777

0.700 to 0.854

0.653 to 0.822

0.738

0.657

0.557 to 0.758

0.547 to 0.737

0.541 to 0.720

0.642
$<0.001$

$<0.001$

0.013

0.004

$<0.001$

$<0.001$

0.002

$\frac{\text { uACR }}{{ }^{a} \text { Compared with 0.5. }}$

0.004

AUC, area under the receiver-operating characteristic curve; CI, confidence interval; u[TIMP2]*[IGFBP7], urinary tissue inhibitor of metalloproteinase-2 and insulin-like growth factor-binding protein 7; uKIM-1, urinary kidney injury moleculer-1; uIL-18, urinary Interleukin-18; uACR, urinary albumin to creatinine ratio. 
Figures

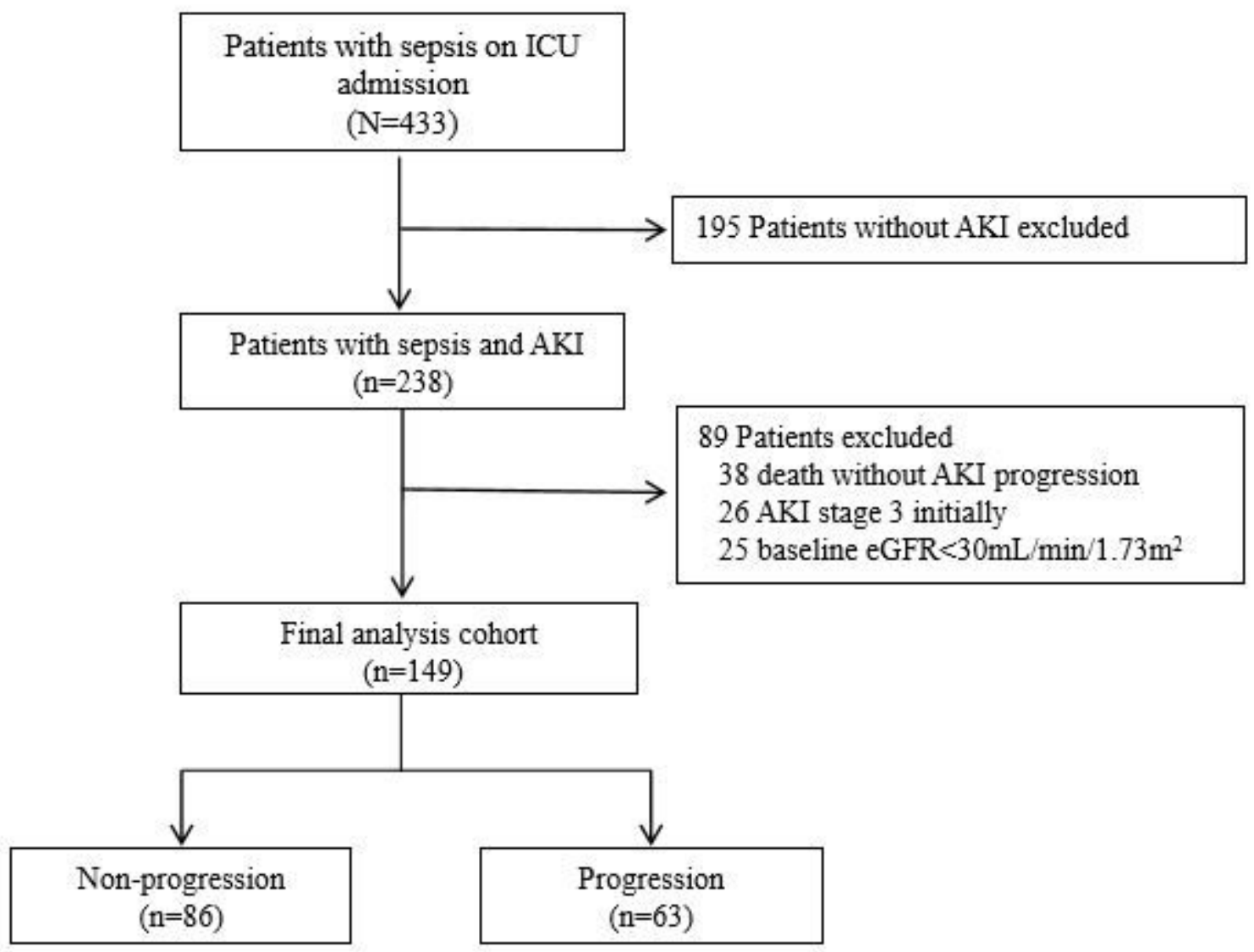

\section{Figure 1}

Flow chart of patient enrollment and exclusion. 

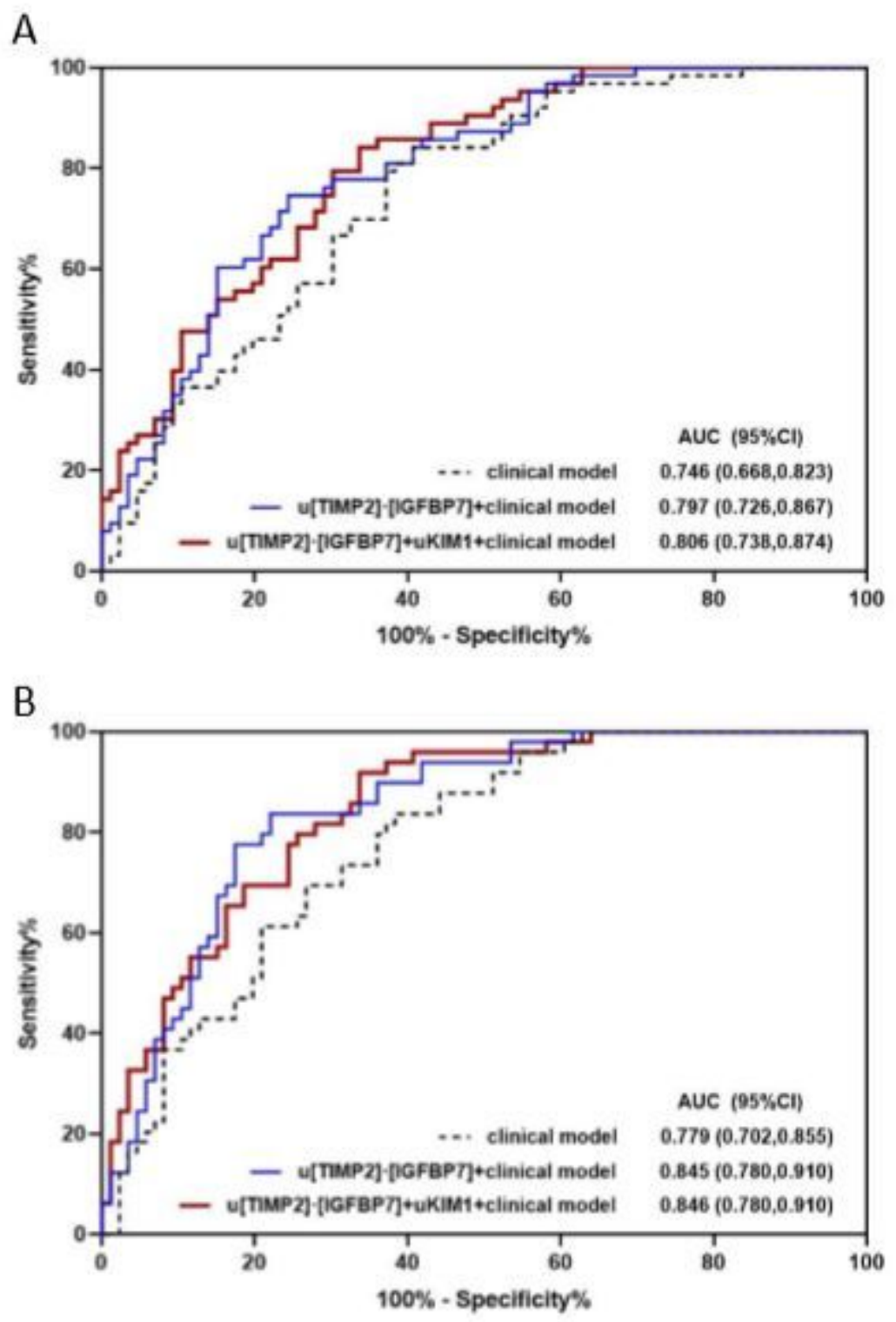

\section{Figure 2}

ROC analyses for predicting AKI progression or AKI progression with death. (A) The AUCs of renal cell arrest and damage biomarkers (UTIMP2*IGFBP7 and uKIM-1), and clinical model, at the time of AKI diagnosis, for predicting AKI progression. (B) The AUCs of renal cell arrest and damage biomarkers (uTIMP2*IGFBP7 and uKIM-1), and clinical model, at the time of AKI diagnosis, for predicting AKI progression with subsequent death. 